\title{
Epidemiología genética de la obesidad: estudios familiares
}

\author{
José Luis Santos $M^{1,2, a}$, José Alfredo Martínez $\mathbf{H}^{\mathbf{2}, \mathrm{b}}$, \\ Francisco Pérez $B^{1}{ }^{1, c}$, Cecilia Albala B ${ }^{1}$. \\ Genetic epidemiology of obesity. \\ Family studies
}

This review focuses on methodological aspects and main results of different family studies that have been conducted to assess the existence of a genetic contribution in human obesity. A genetic component in the etiology of obesity has been elucidated through specific study designs answering different research questions such as: a) Do obesity aggregate in families? b) Is there a genetic contribution to familial clustering? c) Is it possible to localize chromosomal regions that contain susceptibility genes to obesity? d) Is it possible to estimate the risk for developing obesity depending on the genotype profile in candidate genes? There are sufficient evidences indicating the existence of a moderate familial clustering of obesity defined as body mass index $\geq 30$ with a stronger aggregation with more extreme values of body mass index. Twin studies have demonstrated that the familial aggregation of obesity has a genetic component and is not only due to cultural or environmental factors clustered in families. Linkage studies have identified markers and genes related to obesity in virtually all human chromosomes. However, some of these linkage studies have produced conflicting results. Discordant results are even more pronounced in case-control studies that evaluate the association between alleles at candidate genes and obesity. Topics related to study design will acquire increasing importance in order to avoid methodological problems related to trait definition, sample sizes, population stratification by ethnicity and other confounding factors (Rev Méd Chile 2005; 133: 349-61).

(Key Words: Alleles; Body weight; Ethnicity; Genes; Obesity)

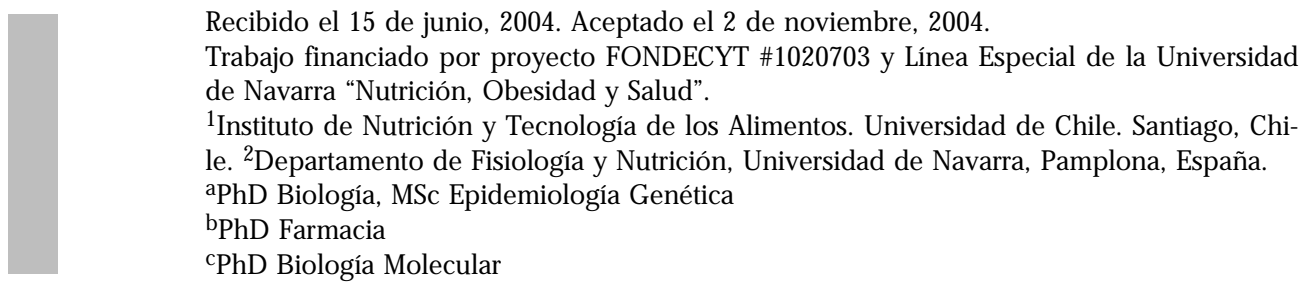

L a obesidad es una condición desfavorable de salud, que se caracteriza por un incremento excesivo de la grasa corporal, originado por un balance energético positivo mantenido en el tiempo ${ }^{1}$. En muchos países, la prevalencia de obesidad ha aumentado notablemente, debido a

Correspondencia a: José Luis Santos. Instituto de Nutrición y Tecnología de los Alimentos, Universidad de Chile. El Líbano 5524. Casilla 138-11, Santiago. Chile. Teléfono: (56-2) 6781456. Fax: (56-2) 2214030. E-mail: jsantos@inta.cl cambios sociales que han llevado a una mayor disponibilidad de alimentos, así como un progresivo descenso de la actividad física ${ }^{2}$. La obesidad constituye un factor de riesgo frente a numerosas enfermedades crónicas, siendo una condición susceptible de experimentar un alivio mediante la alteración adecuada de hábitos de alimentación/ actividad física ${ }^{3}$.

Existen suficientes evidencias que indican que la acumulación de grasa corporal tiene una base genética ${ }^{4}$, no sólo en los casos evidentes de 
formas monogénicas de obesidad ${ }^{5}$, sino también en la obesidad común ${ }^{6}$. Se han descrito más de 430 genes, marcadores genéticos o regiones cromosómicas ligadas a rasgos relacionados con obesidad $^{7,8}$. En esta revisión, examinaremos los estudios familiares utilizados para evaluar la contribución de los factores genéticos en la etiología de la obesidad común, deteniéndonos especialmente en los aspectos metodológicos. Nos centraremos fundamentalmente en los estudios que caracterizan el Índice de Masa Corporal $(\mathrm{IMC}=$ Peso en $\mathrm{kg}$ dividido por la estatura en metros al cuadrado) o el Porcentaje de Grasa Corporal (PGC), sin considerar otros rasgos relacionados, como distribución de grasa corporal o componentes del síndrome metabólico múltiple.

El estudio del componente genético en la obesidad podría enfocarse a través de la formulación secuencial de preguntas, que pueden ser respondidas en el contexto de diferentes estudios epidemiológicos $^{9}$. Un recuento no exhaustivo de preguntas secuenciales de investigación podría ser: 1. ¿Se agrupa la obesidad en familias? 2. ¿Existe un componente genético en esta agregación familiar? 3. ¿Es posible localizar regiones cromosómicas que contienen genes de susceptibilidad frente a la obesidad, mediante la observación de marcadores genéticos en familias? 4. ¿Es posible cuantificar el riesgo de desarrollar obesidad asociado a alelos de susceptibilidad en genes candidatos?

\section{¿SE AGRUPA LA OBESIDAD EN FAMILAS?}

Un primer acercamiento al estudio de la agregación familiar, se refiere a la estimación del riesgo de recurrencia de la enfermedad en familiares de casos índice afectados con la enfermedad. En rasgos complejos como la obesidad, el riesgo de recurrencia en familiares de afectados no sigue patrones mendelianos simples. Una manera de evaluar si la agregación familiar de la obesidad es superior a la esperada, es a través del cálculo de la estadística Lambda-r $(\lambda r)$, donde la letra " $r$ " se refiere a la palabra inglesa relatives. Esta estadística se basa en una idea simple: si existe agregación familiar, el riesgo de desarrollar un rasgo patológico en familiares de casos índice (propositi) afectados con la enfermedad, debe ser superior al riesgo de enfermar en la población general. La estadística $\lambda r$ se define como el cociente de estos dos riesgos. Existe un $\lambda r$ para la evaluación de cada tipo de familiares, y en general, la estadística más usada es $\lambda$-siblings $(\lambda s)$, que se refiere al valor de lambda al evaluar hermanos de casos índice. En enfermedades complejas, existe un amplio rango de valores de $\lambda$ s que oscilan entre $\approx 15$ para la diabetes tipo 1 , hasta valores $\approx 10$ en esquizofrenia y valores más modestos en la enfermedad de Alzheimer de diagnóstico tardío $^{10}$. En contraste, los valores de $\lambda s$ en enfermedades mendelianas simples son mucho mayores, dado que presentan una evidente agregación familiar unida a una muy baja frecuencia de la enfermedad en la población. En rasgos complejos con agregación familiar, los valores de $\lambda \mathrm{r}$ podńan decrecer a medida que se evalúan relaciones familiares más distantes. En este sentido, el examen del patrón de decrecimiento del valor de $\lambda r$ ha servido para comparar la plausibilidad de diferentes modelos de herencia de la enfermedad ${ }^{11}$. Por otra parte, el cálculo del poder estadístico y tamaños muestrales de estudios de ligamiento está basado en los valores de $\lambda \mathrm{r}^{11,12}$.

Estimaciones de $\lambda$ s para obesidad en distintas poblaciones han sido revisadas por Allison et $\mathrm{al}^{12}$ y Bulik y Allison ${ }^{13}$. Para una definición de obesidad como IMC $\geq$ percentil 90 , las estimaciones puntuales de $\lambda$ s oscilan entre 1,5-3,5 según la población estudiada, mientras que estas estimaciones varían entre 2,9 y 6,2 para obesidad definida como IMC $\geq$ percentil 95 en la población. Otros autores han calculado una estadística similar a $\lambda \mathrm{r}$ que es el llamado riesgo relativo estandarizado (RRE) que resulta de estandarizar $\lambda r$ por edad $y$ sexo. Usando familiares de primer grado, Lee et $\mathrm{al}^{14}$, calcularon un RRE para IMC $\geq 30$ cercano a 2 y un RRE para IMC $\geq 45$ de aproximadamente 8 . En un estudio canadiense ${ }^{15}$, se calculó que el RRE para obesidad clase I era sólo de 1,4, mientras que el RRE de obesidad clase II era de 7,1 en familiares en primer grado de casos índice obesos.

En un estudio teórico ${ }^{16}$, se demostró que era improbable esperar una fuerte agregación familiar que fuera únicamente debida a factores ambientales de riesgo reunidos en familias. Por otro lado, se ha indicado que pueden existir errores en la estimación de $\lambda$ s debido al uso de diferentes esquemas de selección o sesgos de información ${ }^{17}$. Por otro lado, se ha demostrado que $\lambda r$ puede expresarse en función del coeficiente de correlación entre el IMC de distintos pares de familiares (por ejemplo: proge- 
nitores-hijos, hermanos-hermanos, tíos-sobrinos $\mathrm{u}$ otros) y la prevalencia de obesidad ${ }^{12}$. En este sentido, las correlaciones entre familiares varían según el tipo de relación familiar, encontrándose correlaciones entre progenitores-hijos en el rango de 0,10-0,35; hermanos de 0,17-0,40; tíos-sobrinos de 0,08-0,14; abuelos-nietos de 0,05-0,0712.

Se ha observado que la obesidad de los padres es un importante factor de riesgo para el desarrollo futuro de la obesidad en la descendencia ${ }^{18}$. Dado que la historia familiar de obesidad reúne tanto factores genéticos de riesgo como factores ambientales o culturales familiares, se ha planteado la utilidad de este índice en estrategias de Salud Pública que buscan intervenciones especiales basadas en personas con alto riesgo de desarrollar rasgos patológicos ${ }^{19}$.

\section{¿EXISTE UN COMPONENTE GENÉTICO EN LA AGRUPACIÓN FAMILIAR DE LA OBESIDAD?}

La comparación de la similitud de pares de gemelos monozigóticos (MZ) y gemelos dizigóticos (DZ), es el acercamiento habitual para distinguir entre la diferente contribución de la genética y del ambiente en el estudio de la agregación familiar. La idea subyacente en los estudios de gemelos MZ y DZ, es que si dos individuos son genéticamente similares, entonces también debenían ser fenotípicamente similares. En este sentido, los gemelos MZ son genéticamente idénticos (salvo un reducido nzúmero de modificaciones postzigóticas), mientras que los gemelos dizigóticos son genéticamente como dos hermanos normales ${ }^{10}$. De este modo, la existencia de un mayor grado de similitud en gemelos MZ con respecto a la correlación en gemelos $\mathrm{DZ}\left(\mathrm{r}_{\mathrm{MZ}}>\mathrm{r}_{\mathrm{DZ}}\right)$ indicanía que la agregación familiar de IMC se encuentra parcialmente bajo control genético ${ }^{20}$. El supuesto crucial en este tipo de estudios, es que tanto los gemelos MZ como los gemelos DZ comparten los factores ambientales de riesgo con el mismo grado de intensidad. Sin embargo, el supuesto de "igualdad de ambiente" podría estar afectado si la correlación ambiental en gemelos MZ es superior a la de los gemelos $\mathrm{DZ}^{21}$, lo que invalidaría en parte las conclusiones derivadas de los estudios de pares de gemelos, al sobreestimar la heredabilidad del rasgo estudiado. Tras la revisión cuantitativa de diferentes estudios de gemelos ${ }^{22}$, se calculó una correlación ponderada para el IMC de
0,74 en gemelos MZ y de 0,32 en gemelos DZ. Con el fin de visualizar esta relación, la Figura 1 muestra los coeficientes de correlación intraclase de simulaciones computacionales del IMC en pares de gemelos de acuerdo a estos cálculos. Adicionalmente, la Tabla 1 muestra las comelaciones para el IMC o PGC estimadas en diferentes estudios de gemelos ${ }^{23-29}$, con referencia especial a un reciente estudio comparativo que reúne datos de aproximadamente 37.000 pares de gemelos de ocho países ${ }^{27}$.

En estudios de pares de gemelos MZ y DZ, es posible obtener una estimación de la heredabilidad, que es la proporción de la variabilidad fenotípica de un rasgo que es explicada por los factores genéti$\cos ^{30}$. Existe un gran número de estudios de gemelos que estiman la heredabilidad de IMC en un rango entre 50-80\% ${ }^{25,31-34}$. El examen de pares de gemelos MZ que han crecido en ambientes diferentes, ofrece la oportunidad de medir directamente la heredabilidad del IMC sin necesidad de gemelos DZ, encontrándose valores de heredabilidad similares a los estimados mediante la comparación de gemelos MZ y DZ ${ }^{35,36}$. Por otro lado, los estudios de correlación de familiares también permiten estimar la heredabilidad del IMC, habiéndose obtenido estimaciones de la heredabilidad sensiblemente inferiores (25-40\%) a las calculadas en estudios de gemelos. Maes et $\mathrm{al}^{22}$, discuten las estimaciones de la heredabilidad obtenidas en estudios que usan diferentes tipos de familiares y concluye que los estudios de gemelos ofrecen un mayor control del efecto de variables como la edad y otras variables asociadas con la edad. Es importante resaltar que la heredabilidad es dependiente de cada población y que la estimación de altos valores de heredabilidad no restan importancia al efecto de factores nogenéticos, como queda de manifiesto al contrastar la alta heredabilidad del IMC con la evidente importancia de factores sociales en la obesidad.

Un tipo de estudio de gemelos diferente al discutido en el párrafo anterior, hace referencia a los experimentos de balance energético positivo y negativo basados únicamente en pares de gemelos $\mathrm{MZ}$, que son sometidos a diferentes condiciones de dieta y ejercicio físico, que favorecían el balance energético positivo 0 negativo ${ }^{37}$. Estos estudios plantean que la ganancia 0 pérdida de peso es dependiente del genotipo, dado que la variabilidad intra-par de gemelos en la ganancia/pérdida de peso, es menor que la variabilidad entre diferentes 
pares de gemelos MZ. Por otro lado, los estudios de adoptados han encontrado una mayor correlación en el índice de masa corporal entre los hijos y sus padres biológicos que entre los hijos y sus padres adoptivos, lo que indicaría la presencia de un importante componente genético en el IMC ${ }^{38}$.

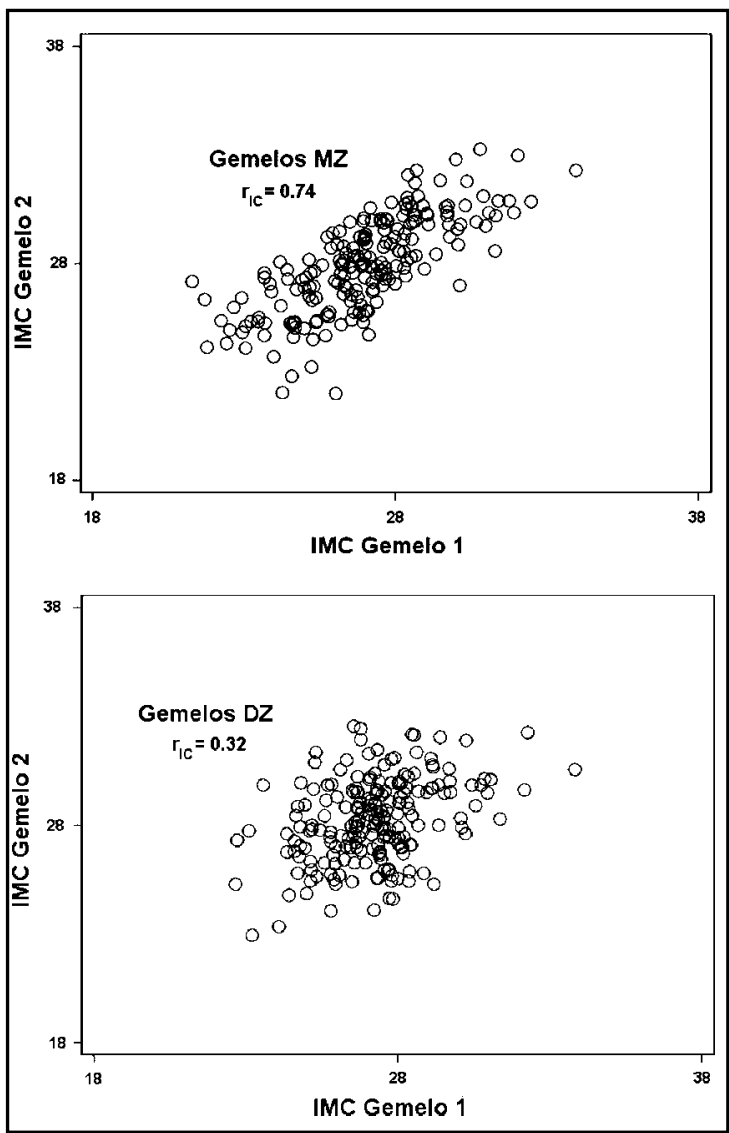

FiguRA 1. Correlación simulada entre pares de gemelos monozigóticos (MZ) y dizigóticos (DZ) para el Índice de Masa Corporal (IMC).

Los datos de IMC en gemelos fueron obtenidos mediante simulaciones computacionales en 200 pares MZ y 200 pares DZ con distribuciones normales bivariadas con coeficientes de correlación intraclase $\left(r_{\mathrm{IC}}\right)$ de 0,74 en gemelos MZ y 0,32 en gemelos DZ, de acuerdo a la revisión de Maes et $\mathrm{al}^{22}$. La razón de usar $\mathrm{r}_{\mathrm{IC}}$ en lugar de coeficientes de correlación de Pearson es que la asignación de un par de gemelos al eje de abcisas u ordenadas es arbitraria. Las simulaciones fueron realizadas con el programa STATA 8.2 (Statacorp, Collage Station, USA, 2004).
ES POSIBLE LOCALZAR REGIONES CROMOSÓMICAS QUE CONTIENEN GENES DE SUSCEPTIBILIDAD FRENTE A LA OBESIDAD?

El objetivo de los estudios de ligamiento es la localización de regiones cromosómicas que contengan genes de susceptibilidad frente a enfermedades. El término "ligamiento" se refiere a la cercanía física entre loci genéticos (medida como $\Theta=$ frecuencia de recombinación) que oniginańa la cosegregación de marcadores genéticos anónimos y genes de susceptibilidad. En un típico estudio de ligamiento de genoma completo (bamido genómico), se generan genotipos de 300-500 marcadores microsatélite de alta heterocigosidad espaciados cada 5-10 centimorgans a lo largo del genoma. Los estudios de ligamiento han sido muy exitosos en la identificación de genes relacionados con enfermedades mendelianas en familias multigeneracionales ${ }^{39}$. Sin embargo, estos estudios no han sido tan efectivos en el estudio de enfermedades complejas ${ }^{40}$, donde las penetrancias son generalmente desconocidas y el análisis de ligamiento se realiza en unidades familiares simples como los pares de hermanos afectados y sus progenitores. En rasgos cualitativos, un exceso de alelos idénticos por descendencia señalanía la posible existencia de genes de susceptibilidad en la región cromosómica delimitada por marcadores genéticos ${ }^{41}$. En rasgos cuantitativos como el IMC, el enfoque de análisis de ligamiento más intuitivo se basa en la regresión de una medida de similitud/discordancia del IMC para cada par de hermanos (por ejemplo, el cuadrado de la diferencia entre hermanos) versus el número de alelos idénticos por descendencia en un marcador dado ${ }^{42}$. Se concluye con la existencia de ligamiento en una región cromosómica dada cuando existe concurrencia entre una mayor similitud fenotípica y un exceso en la proporción de alelos idénticos por descendencia. En este sentido, las evidencias a favor de ligamiento se evalúan mediante la estadística LOD score que es el logaritmo en base 10 del cociente de verosimilitudes que compara los datos bajo la hipótesis de ligamiento $(\Theta<0,5)$ con respecto a la hipótesis de inexistencia de ligamiento $(\Theta=0,5)$. Cuanto más cercano es un marcador anónimo al gen de susceptibilidad, mayor es la cosegregación entre ambos loci, menor es $\Theta$, y mayor es el valor del LOD score ${ }^{43}$. En enfermedades mendelianas, un LOD score superior a 3 es generalmente aceptado como suficiente evidencia para aceptar la existencia de ligamiento de un marcador con el gen responsable de la enfermedad. En rasgos complejos, existen referencias de valores de 
LOD score para establecer el peso de las evidencias científicas que lleva a establecer ligamiento ${ }^{44}$.

Se han efectuado más de 30 barridos genómicos sobre obesidad en familias provenientes de diferentes grupos étnicos, encontrándose evidencias de ligamiento virtualmente en todos los cromosomas 7,45 . Resultados positivos de algunas regiones cromosómicas han sido replicados en diferentes estudios (por ejemplo, 1p36, 3q27, 7q34, 10p12, 11q22-24, 12p12, 20q entre otras). Entre los aspectos novedosos de los estudios de ligamiento publicados durante los primeros meses de 200446-51, cabe destacar un artículo que considera mediciones seriadas de IMC en un seguimiento promedio de 20,2 años, encontrando ligamiento significativo en marcadores del cromosoma 12 (LOD score $=3,0$ ), y valores que sugieren la existencia de ligamiento (LOD score 2,2-3,5) en marcadores de los cromosomas 5 y 7. Por otro lado, otro estudio se ha centrado en rasgos específicos de ingesta energética, encontrando ligamiento significativo de estas variables para las regiones 1p21.2, 20q13.13 y 12q14.151. Finalmente, los estudios de ligamiento en enfermedades complejas han tenido una revitalización reciente de la mano de métodos estadísticos que evalúan no sólo hermanos, sino que hacen uso de la información de otras relaciones familiares ${ }^{52}$. En este sentido, algunos estudios como QFS (Qué bec Family Study), HERTAGE (Health, Risk, Exercise Training and Genetics) y SAFHS (San Antonio

Tabla 1. Correlaciones entre gemelos M Z y D Z para el Índice de M asa Corporal (IM C) o el Porcentaje de Grasa C orporal (PGC) en estudios seleccionados

\begin{tabular}{|c|c|c|c|c|c|c|c|}
\hline Referencia & $\begin{array}{l}\text { Edad } \\
\text { (años) }\end{array}$ & Población & Rasgo & $\begin{array}{l}\text { Muje } \\
\text { Gemelos } \\
\text { MZ }\end{array}$ & $\begin{array}{l}\text { eres } \\
\text { Gemelos } \\
\text { DZ }\end{array}$ & $\begin{array}{l}\text { Hon } \\
\text { Gemelos } \\
\text { MZ }\end{array}$ & $\begin{array}{l}\text { Gemelos } \\
\text { DZ }\end{array}$ \\
\hline $\begin{array}{l}\text { Pietlainen et al } \\
\text { (1999) }\end{array}$ & 17 & Finlandia & IMC & 0,85 & 0,41 & 0,86 & 0,37 \\
\hline $\begin{array}{l}\text { Korkeila et al } \\
\text { (1996) }\end{array}$ & $60-76$ & Finlandia & IMC & 0,74 & 0,41 & 0,51 & 0,33 \\
\hline $\begin{array}{l}\text { Stunkard et al } \\
\text { (1986) }\end{array}$ & $40-50$ & USA & IMC & - & - & 0,81 & 0,42 \\
\hline $\begin{array}{l}\text { Schousbe et al } \\
\text { (2004) }\end{array}$ & $30-39$ & Dinamarca & IMC & 0,72 & 0,33 & 0,67 & 0,32 \\
\hline $\begin{array}{l}\text { Schousbe et al } \\
\text { (2004) }\end{array}$ & $30-39$ & Dinamarca & IMC & 0,73 & 0,35 & 0,77 & 0,35 \\
\hline $\begin{array}{l}\text { Schousbe et al } \\
\text { (2004) }\end{array}$ & $30-39$ & Finlandia & IMC & 0,78 & 0,37 & 0,74 & 0,32 \\
\hline $\begin{array}{l}\text { Schousbe et al } \\
\text { (2004) }\end{array}$ & $30-39$ & Italia & IMC & 0,83 & 0,58 & 0,83 & 0,52 \\
\hline $\begin{array}{l}\text { Schousbe et al } \\
\text { (2004) }\end{array}$ & $30-39$ & Holanda & IMC & 0,79 & 0,41 & 0,65 & 0,31 \\
\hline $\begin{array}{l}\text { Schousbe et al } \\
\text { (2004) }\end{array}$ & $30-39$ & Noruega & IMC & 0,74 & 0,35 & 0,69 & 0,41 \\
\hline $\begin{array}{l}\text { Schousbe et al } \\
\text { (2004) }\end{array}$ & $30-39$ & Suecia & IMC & 0,73 & 0,36 & 0,77 & 0,35 \\
\hline $\begin{array}{l}\text { Nelson et al } \\
\text { (2002) }\end{array}$ & $22-88$ & USA $^{\#}$ & IMC & 0,66 & 0,48 & 0,84 & 0,40 \\
\hline $\begin{array}{l}\text { Schousbe et al } \\
\text { (2003) }\end{array}$ & $18-67$ & Dinamarca & PGC & 0,72 & 0,42 & 0,65 & 0,33 \\
\hline $\begin{array}{l}\text { Samaras et al } \\
\text { (1997) }\end{array}$ & $55-65$ & USA & PGC & 0,77 & 0,53 & - & - \\
\hline
\end{tabular}

*No se muestran las correlaciones en gemelos DZ de sexo opuesto. \#Población afroamericana. 
Family Heart Study) han sido utilizados tanto para la evaluación de agregación familiar como para la búsqueda de genes relacionados con la obesidad ${ }^{53}$. La Figura 2 muestra algunas regiones cromosómicas ligadas con la obesidad y que han sido identificadas a través de al menos dos estudios de ligamiento independientes ${ }^{45}$.

¿ES POSIBLE CUANTIFICAR EL RIESGO DE DESARROLLAR OBESIDAD ASOCIADO A CIERTOS POLMORFISMOS GENÉTICOS?

La Figura 3 muestra algunos de los genes que han sido relacionados con la obesidad y que han sido identificados a través de diferentes tipos de estu$\operatorname{dios}^{8}$. En algunos casos, la posición de estos genes coincide con regiones previamente delimitadas en estudios de ligamiento, lo que reforzaría su posible participación como genes de susceptibilidad frente a la obesidad. Gran parte de los estudios que evalúan el riesgo asociado a polimorfismos genéticos en genes candidatos y su relación con la obesidad u otras enfermedades de etiología multifactorial se ha llevado a cabo a través de estudios de caso-control ${ }^{54,55}$. Ha existido una enorme discordancia en los resultados obtenidos en estos estudios al evaluar diferentes poblaciones ${ }^{56}$. En este tipo de estudios, asociaciones positivas entre polimorfismos genéticos y enfermedades serían debidas básicamente a alguna de las siguientes circunstancias ${ }^{57}$ : a) efecto causal del alelo estudiado; b) asociación de tipo falso positivo debido a errores aleatorios de muestreo; c) desequilibrio de ligamiento con un alelo del verdadero gen de susceptibilidad; o d) sesgo sistemático en el diseño del estudio, como los que generan efectos de confusión originados por la estratificación genética por etnia en la población ${ }^{58}$.

Con el fin de evitar asociaciones no causales gen-enfermedad que sean debidas a la estratifica-

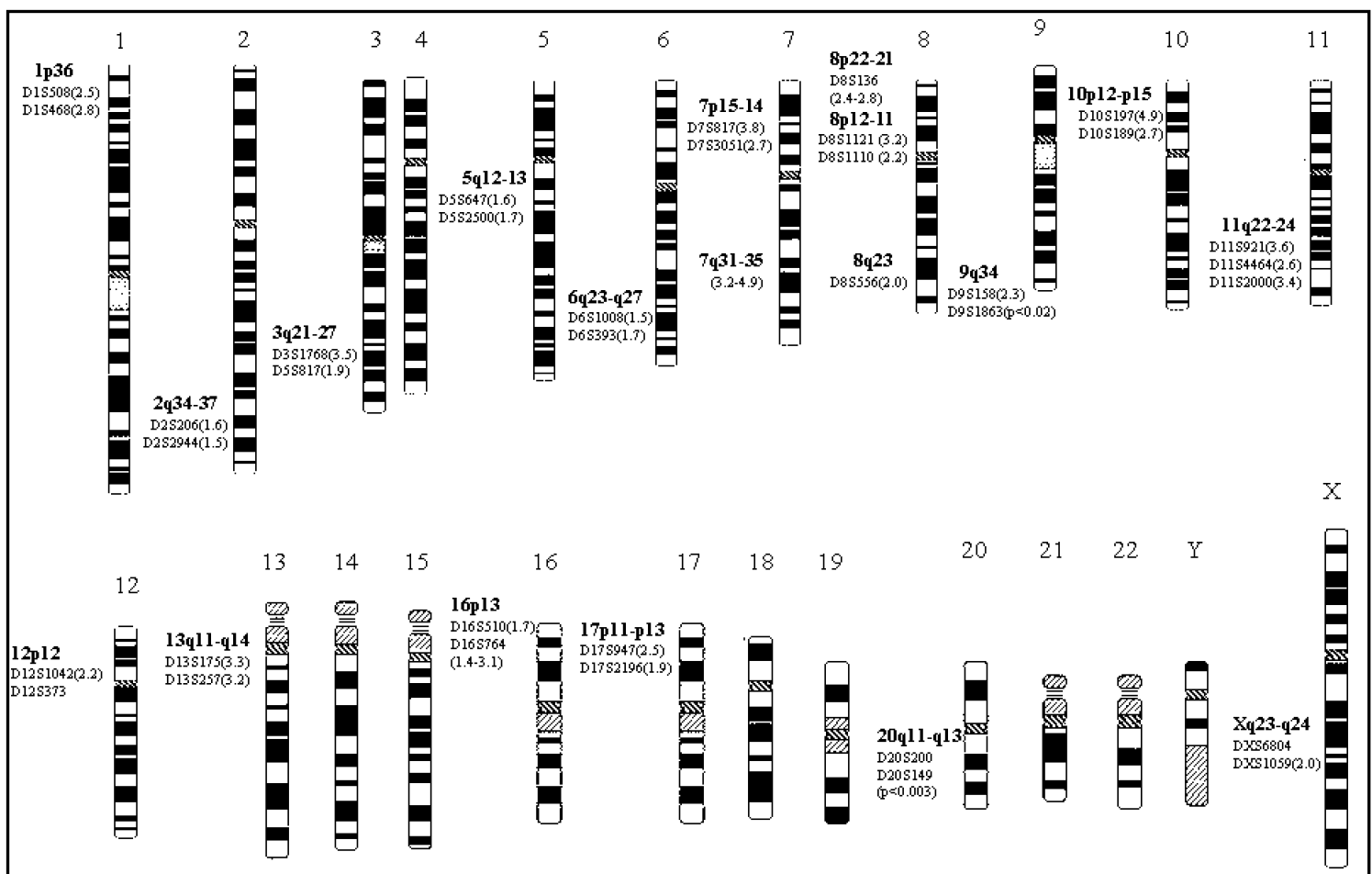

FIGURA 2. Localización aproximada de algunas regiones cromosómicas relacionadas con la obesidad en estudios de ligamiento.

Las regiones cromosómicas que se señalan fueron seleccionadas de acuerdo a su identificación en al menos dos estudios de ligamiento independientes, según la revisión de Damcott et $\mathrm{al}^{45}$. Se indica el nombre de los marcadores microsatélite utilizados en los estudios de ligamiento junto con el valor de LOD score o la significación estadística ( $p$-value, entre paréntesis). 


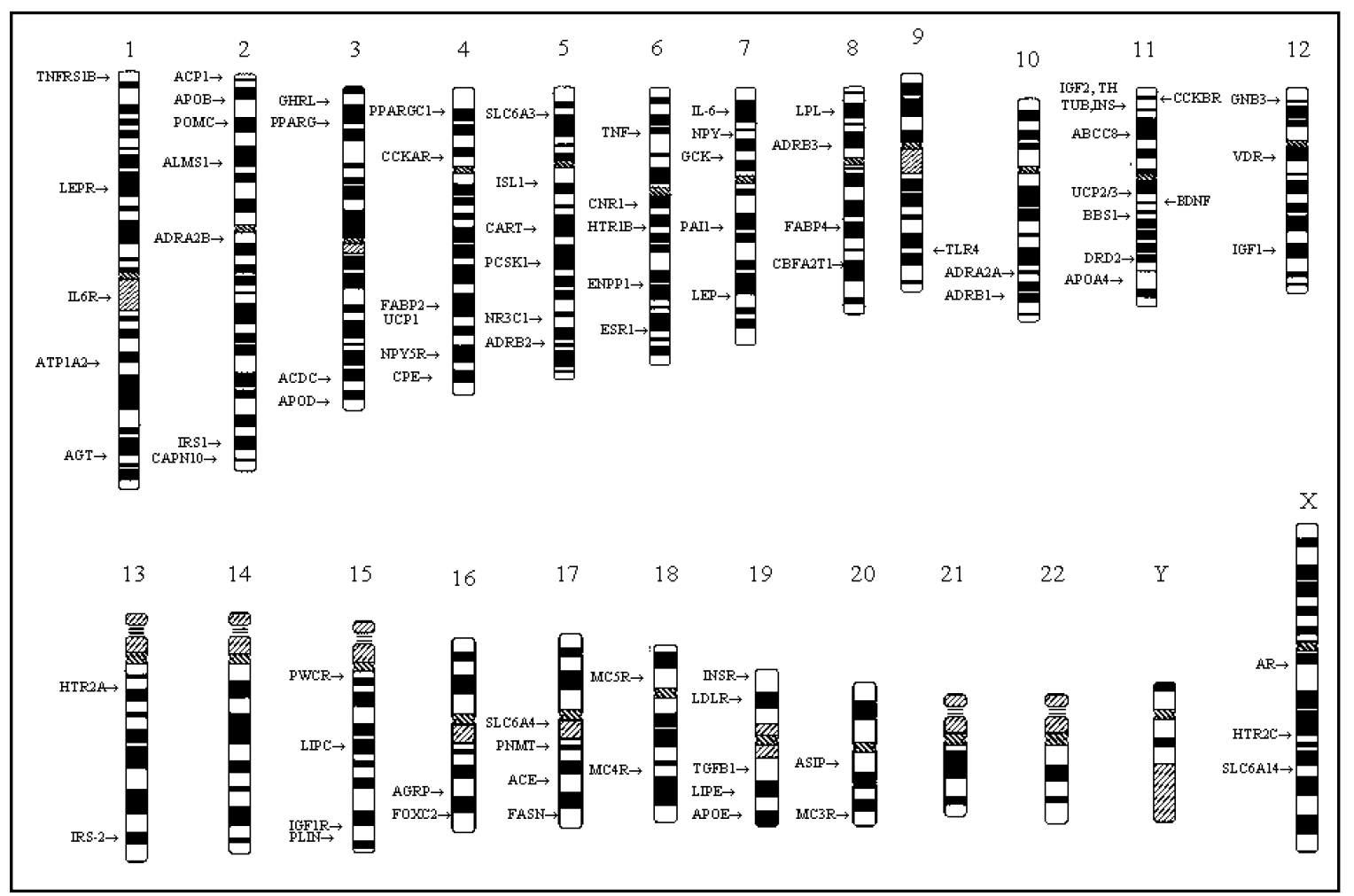

FiguRA 3. Localización cromosómica aproximada de algunos genes relacionados con la obesidad y sus comorbilidades.

Cromosoma 1: TNFRSF1B: Miembro 1B de la superfamilia de receptores del factor de necrosis tumoral (Receptor 2 del factor de necrosis tumoral TNFR-2); LEPR: Receptor de Leptina; IL6R: Receptor de intenleuquina 6; ATP1A2: Polipéptido alfa2 (+) de la ATPasa $\mathrm{Na}^{+} / \mathrm{K}^{+}$; AGT: Angiotensina I; Cromosoma 2: ACP1: Fosfatasa ácida 1; APOB: Apolipoproteína B; POMC: Proopiomelanocortina; ALMS1: Locus 1 del síndrome de Alstrom. ADRA2B: Receptor adrenérgico alfa 2B; IRS1: Sustrato insulínico 1; CAPN10: Calpain-10; Cromosoma 3: GHRL: Precursor de ghrelina; PPARG: Receptor gamma activado por la proliferación de peroxisomas; ACDC: Adiponectina (APM1); APOD: Apolipoproteína D; Cromosoma 4: PPARGC1: Coactivador 1 del receptor gamma activado por la proliferación de peroxisomas; CCKAR: Receptor A de la colecistoquinina; FABP2: Proteína ligante de ácidos grasos 2 (intestinal); UCP-1: Proténa desacoplante- 1; NPY5R: Receptor 5 del neuropéptido Y; CPE: Carboxipeptidasa E; Cromosoma 5: SLC6A3: Miembro 3 de la familia 6 de portadores de solutos; ISL1: Factor de transcripción ISL1; CART: cocaine and amphetamineregulated transcript; PCSK1: Prohormona convertasa 1; NR3C1: Receptor de glucocorticoides; ADRB2: Receptor beta2 adrenérgico; Cromosoma 6: TNF: Factor alfa de necrosis tumoral; CNR1: cannabinoid receptor 1; HTR1B: Receptor 1B de serotonina; ENPP1: Plasma-cell membrane glycoprotein-1 (PC-1); ESR1: Receptor de estrógenos 1 ( $\alpha$ ); Cromosoma 7: IL6: Intenleuquina 6; NPY: Neuropéptido Y; GCK: glucoquinasa; PAI1: plasminogen activator inhibitor - 1; LEP: Leptina; Cromosoma 8: LPL: Lipoproteína lipasa; ADRB3: Receptor beta3 adrenérgico; FABP4: Proteína ligante de ácidos grasos 2 (adipocitaria); CBFA2T1: Factor de transcripción MTG8; Cromosoma 9: TLR4: Toll-like receptor 4; Cromosoma 10: ADRA2A: Receptor adrenérgico alfa 2A; ADRB1: Receptor beta1 adrenérgico; Cromosoma 11: INS: Insulina; IGF-2: Factor de crecimiento semejante a la insulina- 2; TH: Tirosina hidroxilasa; TUB: Homólogo del gen Tubby; CCKBR: Receptor B de colecistoquinina; ABCC8: Receptor de sulfonilurea (SUR); UCP-2/3: Proteínas desacoplantes 2 y 3; BDNF: brain-derived neurotrophic factor; BBS1: Locus 1 del síndrome de Bardet-Biedl; DRD2: Receptor D2 de dopamina; APOA4: Lipoproteína A-IV; Cromosoma 12: GNB3: Subunidad beta 3 de la proteína G; VDR: Receptor de la vitamina D; IGF-1: Factor de crecimiento semejante a la insulina- 1; Cromosoma 13: HTR2A: Receptor 2A de la serotonina; IRS2: Sustrato insulínico- 2; Cromosoma 15: PWCR: Región cromosomal de Prader-Willi; LPC: Lipasa hepática; IGF1R: Receptor del factor de crecimiento semejante a la insulina - 1. PUN: Perilipina. Cromosoma 16: AGRP: Proteína relacionada con agouti; FOXC2: Forkhead transcription factor FoxC2; Cromosoma 17: SLC6A4: Miembro 4 de la familia 6 de portadores de solutos; PNMT: Phenylethanolamine N-methyltransferase; ACE: Enzima convertidora de angiotensina; FASN: Sintetasa de ácidos grasos; Cromosoma 18: MC5R: Receptor de melanocortina 5; MC4R: Receptor de melanocortina 4; Cromosoma 19: INSR: Receptor de insulina; LDR: Receptor de lipoproteína de baja densidad; TGFB1: Factor B1 de crecimiento transformante; LPE: Lipasa sensible a homonas; Cromosoma 20: ASIP: Proteína señalizadora de Agouti; MC3R: Receptor de melanocortina 3; Cromosoma X: AR: receptor de andrógenos; HTR2C: Receptor 2C de serotonina; SLC6A14: Miembro 14 de la familia 6 de portadores de solutos. El mapa muestra localizaciones aproximadas de los genes. Ver también el mapa genético de la obesidad (Snyder et al. 2004) que se encuentra disponible en: htpp:/l obesitygene.pbrc.edu. Nomenclatura de genes obtenida en: http://www.ncbi.nlm.nih.gov/ 
ción genética, se ha extendido el uso de diseños de estudio insensibles a este efecto de confusión, como el diseño de tríos de casos-progenitores $^{59,60}$. En su enfoque más clásico, se seleccionan familias a través de un caso índice afectado, y a partir de ese caso, se obtienen los genotipos de los dos progenitores. La asociación entre el factor genético y enfermedad origina que, en estas familias, la transmisión de alelos desde padres heterocigotos hacia sus hijos enfermos se aparten del valor esperado bajo la hipótesis nula de noasociación (probabilidad de transmisión $=50 \%$ ). La transmisión alélica se evalúa mediante "Transmisión Disequilibrium Test" (TDT) y otros métodos relacionados ${ }^{61,62}$. En el análisis de estos tríos, las desviaciones en la transmisión del alelo de susceptibilidad dependen del riesgo relativo genotípico. Por tanto, el análisis de la transmisión de alelos en tríos de casos-progenitores permite estimar la magnitud del riesgo asociado a ciertos alelos ${ }^{63}$.

En el caso de rasgos cuantitativos, existen dos acercamientos diferentes en los estudios de tríos casos-progenitores. Por un lado, hay estudios en los que la selección de familias se hace a través de casos índice con valores de IMC que superen cierto umbral (por ejemplo, IMC $\geq 30$ ) y la posterior incorporación de los progenitores en un análisis clásico de TDT. Por otro lado, existe la posibilidad de utilizar extensiones del TDT que evalúan rasgos cuantitativos en un grupo de estadísticas llamadas QTDT ("quantitativeTDT") ${ }^{64}$. También se han propuesto extensiones de TDT para rasgos cuantitativos mediante el uso de regresión logística ${ }^{65}$ en la que se modela la probabilidad de transmisión de alelos de susceptibilidad (p) en función de los valores de una variable continua (por ejemplo, IMC).

$$
\operatorname{Ln}[\mathrm{p} /(1-\mathrm{p})]=\alpha+\beta(\mathrm{IMC})
$$

Para algunos investigadores, pudiera parecer extraño modelar la transmisión alélica como variable dependiente y el IMC como variable independiente y no al revés. Sin embargo, esta estrategia enfatiza que la distorsión en la segregación alélica ocurre sólo como consecuencia de la selección sesgada de personas con valores extremos del rasgo en estudio debido a la presencia de asociación genotipo-IMC. Es decir, si existe asociación
gen-IMC, la transmisión del alelo de susceptibilidad en tríos por encima de cierto umbral (por ejemplo, IMC $\geq 30$ ) será superior al $50 \%$ (Figura 4 ). Por otro lado, el modelo de regresión logística así expresado permite incluir variables cuantitativas o categóricas que pueden agregarse como factores de confusión o modificadores de efecto. Sin embargo, cuando la selección de familias se realiza independientemente del IMC, la transmisión alélica global será de $50 \%$, siempre bajo el supuesto de inexistencia de distorsión en la segregación alélica durante la meiosis ${ }^{66}$. Existe un número limitado, aunque creciente, de estudios que han publicado asociaciones entre polimorfismos genéticos y variables relacionadas con la obesidad a través del uso de tríos casos-progenitores ${ }^{67-85}$ (Tabla 2).

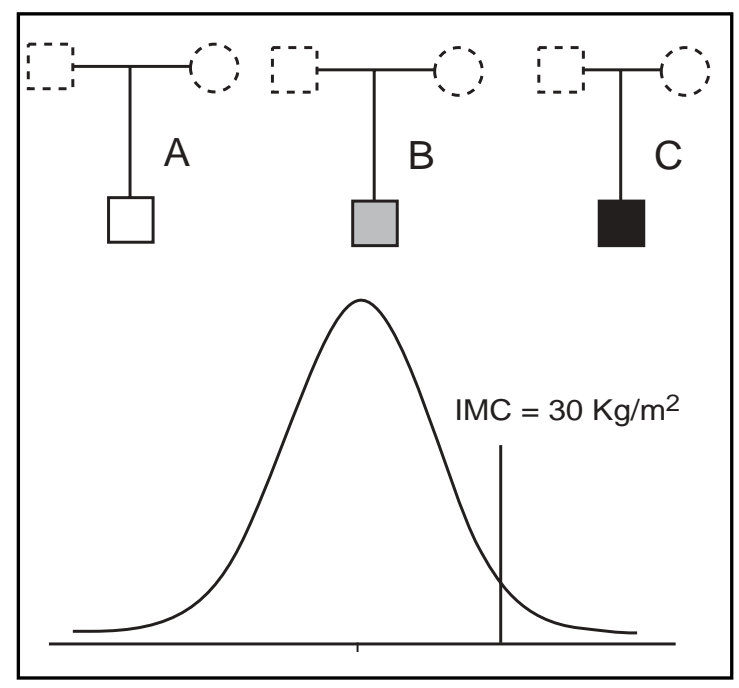

Figura 4. Análisis de transmisión alélica en tríos de hijos-progenitores para rasgos cuantitativos (IMC= Índice de Masa Corporal).

Los símbolos familiares oscuros en los hijos indican la existencia de altos valores de IMC (por ejemplo, IMC $\geq 30$ ), mientras que los símbolos grises y claros representan valores intermedios y bajos de IMC respectivamente. No se indica el IMC de los progenitores. Si existe asociación gen-obesidad, la transmisión del alelo de susceptibilidad en tríos de tipo $\mathrm{C}$ (con valores altos de IMC) será superior al 50\% (enfoque TDT clásico). Si la selección de familias se realiza independientemente del IMC, la transmisión alélica global (tríos de tipo $A+B+C$ ) será de $50 \%$ según indican las leyes de Mendel ${ }^{65,66}$. 
Tabla 2. Estudios de casos - progenitores que evalúan la asociación entre polimorfismos genéticos y obesidad

\begin{tabular}{|c|c|c|}
\hline Referencia & Gen/Región cromosómica/Alelos & Análisis \\
\hline $\begin{array}{l}\text { Hinney et al } \\
\text { (1997) }\end{array}$ & $\begin{array}{l}\text { Polimorfismo Trp64Arg del receptor } \\
\text { beta3 adrenérgico }\end{array}$ & $\begin{array}{l}\text { Enfoque TDT clásico en pacientes } \\
\text { obesos }\end{array}$ \\
\hline $\begin{array}{l}\text { Hinney et al } \\
\text { (1998) }\end{array}$ & Polimorfismos del gen de la leptina & $\begin{array}{l}\text { Enfoque TDT clásico en pacientes } \\
\text { obesos }\end{array}$ \\
\hline $\begin{array}{l}\text { Roth et al } \\
\text { (1997) }\end{array}$ & $\begin{array}{l}\text { Repeticiones de dinucleótidos } \\
\text { D7S504 y D7S1875 }\end{array}$ & $\begin{array}{l}\text { Enfoque TDT clásico en pacientes } \\
\text { obesos }\end{array}$ \\
\hline $\begin{array}{l}\text { Roth et al } \\
\text { (1998) }\end{array}$ & $\begin{array}{l}\text { Marcadores microsatélite del gen del } \\
\text { receptor de leptina }\end{array}$ & $\begin{array}{l}\text { Enfoque TDT clásico en pacientes } \\
\text { obesos }\end{array}$ \\
\hline $\begin{array}{l}\text { Hinney et al } \\
\text { (1999) }\end{array}$ & $\begin{array}{l}\text { Polimorfismos del receptor } 4 \text { de } \\
\text { dopamina }\end{array}$ & $\begin{array}{l}\text { Enfoque TDT clásico en pacientes } \\
\text { obesos }\end{array}$ \\
\hline $\begin{array}{l}\text { Hinney et al } \\
\text { (2000) }\end{array}$ & $\begin{array}{l}\text { Repeticiones de dinucleótidos CA en } \\
\text { 10p13-10ql1 }\end{array}$ & $\begin{array}{l}\text { Enfoque TDT clásico en pacientes } \\
\text { obesos }\end{array}$ \\
\hline $\begin{array}{l}\text { Cassell et al } \\
(2000)\end{array}$ & $\begin{array}{l}\text { Polimorfismos de la proteína } \\
\text { desacoplante } 3\end{array}$ & TDT cuantitativo (QTDT) \\
\hline $\begin{array}{l}\text { Le Stunff et al } \\
\text { (2001) }\end{array}$ & $\begin{array}{l}\text { Repeticiones en tándem del gen de la } \\
\text { insulina }\end{array}$ & $\begin{array}{l}\text { Enfoque TDT en obesos y } \\
\text { transmisión paterna diferencial }\end{array}$ \\
\hline $\begin{array}{l}\text { Delplanque et al } \\
\text { (2003) }\end{array}$ & $\begin{array}{l}\text { Polimorfismos simples del gen de la } \\
\text { urocortina }\end{array}$ & $\begin{array}{l}\text { Enfoque TDT clásico en pacientes } \\
\text { obesos }\end{array}$ \\
\hline $\begin{array}{l}\text { Hinney et al } \\
\text { (2003) }\end{array}$ & $\begin{array}{l}\text { Polimorfismos del gen del receptor } 4 \\
\text { de melanocortina }\end{array}$ & $\begin{array}{l}\text { Enfoque TDT clásico en pacientes } \\
\text { obesos }\end{array}$ \\
\hline $\begin{array}{l}\text { Huang et al } \\
(2003)\end{array}$ & $\begin{array}{l}\text { Repeticiones de dinucleótidos CA en } \\
\text { interleuquina } 6\end{array}$ & TDT cuantitativo (QTDT) \\
\hline $\begin{array}{l}\text { Long et al } \\
\text { (2003) }\end{array}$ & $\begin{array}{l}\text { Polimorfismos simples en los genes } \\
\text { ApoE y TGF-beta1 }\end{array}$ & TDT cuantitativo (QTDT) \\
\hline $\begin{array}{l}\text { Schauble et al } \\
\text { (2003) }\end{array}$ & $\begin{array}{l}\text { Polimorfismo simple de la región } \\
\text { promotora de UCP2 }\end{array}$ & $\begin{array}{l}\text { Enfoque TDT clásico y métodos } \\
\text { relacionados }\end{array}$ \\
\hline $\begin{array}{l}\text { Li et al } \\
(2003)\end{array}$ & $\begin{array}{l}\text { Repeticiones de dinucleótidos CA en } \\
7 \mathrm{q} 22.1-7 q 35\end{array}$ & $\begin{array}{l}\text { QTDT y enfoque TDT clásico en } \\
\text { pacientes obesos }\end{array}$ \\
\hline $\begin{array}{l}\text { Geller et al } \\
\text { (2004) }\end{array}$ & $\begin{array}{l}\text { Alelo I103 del receptor } 4 \text { de } \\
\text { melanocortina }\end{array}$ & $\begin{array}{l}\text { Enfoque TDT clásico en pacientes } \\
\text { obesos }\end{array}$ \\
\hline $\begin{array}{l}\text { Heude et al } \\
\text { (2004) }\end{array}$ & $\begin{array}{l}\text { Repeticiones en tándem del gen de la } \\
\text { insulina }\end{array}$ & $\begin{array}{l}\text { Enfoque TDT en obesos y } \\
\text { transmisión paterna diferencial }\end{array}$ \\
\hline $\begin{array}{l}\text { Camarena et al } \\
\text { (2004) }\end{array}$ & $\begin{array}{l}\text { Polimorfismo EcoRV del gen de la } \\
\text { Monoamino oxidasa A }\end{array}$ & $\begin{array}{l}\text { Enfoque TDT clásico en pacientes } \\
\text { obesos }\end{array}$ \\
\hline $\begin{array}{l}\text { Wang et al } \\
(2004)\end{array}$ & $\begin{array}{l}\text { Polimorfismos simples en el receptor } \\
\text { de la Ghrelina }\end{array}$ & $\begin{array}{l}\text { Enfoque TDT clásico en pacientes } \\
\text { obesos }\end{array}$ \\
\hline $\begin{array}{l}\text { Wang et al } \\
(2004)\end{array}$ & $\begin{array}{l}\text { Alelo I/D de la enzima convertidora } \\
\text { de angiotensina }\end{array}$ & TDT cuantitativo (QTDT) \\
\hline $\begin{array}{l}\text { Jiang et al } \\
\text { (2004) }\end{array}$ & Polimorfismos del gen de la leptina & $\begin{array}{l}\text { Enfoque TDT clásico en pacientes } \\
\text { obesos y TDT cuantitativo }\end{array}$ \\
\hline
\end{tabular}

TDT: Transmission Disequilibrium Test. 


\section{REFERENCIAS}

1. Martínez JA. Body-weight regulation: causes of obesity. Proc Nutr Soc 2000; 59: 337-45.

2. Prentice AM, JebB SA. Obesity in Britain: gluttony or sloth? BMJ 1995; 311: 437-9.

3. Hill JO, Wyatt HR, Reed GW, Peters JC. Obesity and the environment: where do we go from here? Science 2003; 299: 853-5.

4. Loos RJ, Bouchard C. Obesity is it a genetic disorder? J Intern Med 2003; 254: 401-25.

5. O'RahiLy S, Farooqi IS, Yeo GS, Chaшs BG. Minireview: human obesity-lessons from monogenic disorders. Endocrinology 2003; 144: 3757-64.

6. Ochoa M, Marti A, Martínez J. Estudios sobre la obesidad en genes candidatos. Med Clin 2004; 122: 542-51.

7. Snyder EE, Walts B, Perusse L, Chagnon YC, WeisnaGel SJ, Rankinen T et al. The human obesity gene map: the 2003 update. Obes Res 2004; 12: 369-439.

8. Comuzzie AG, Awson DB. The search for human obesity genes. Science 1998; 280: 1374-7.

9. Khoury MJ, Beaty TH, Cohen BH. Fundamentals of Genetic Epidemiology. Oxford University Press. New York 1993.

10. Strachan T, Read AP. Human Molecular Genetics 2. BIOS Scientific Publishers Ltd. Oxford 1999.

11. Rusch N. Linkage strategies for genetically complex traits. I. Multilocus models. Am J Hum Genet 1990; 46: 222-8.

12. Awson DB, Faith MS, Nathan JS. Risch's lambda values for human obesity. Int J Obes Relat Metab Disord 1996; 20: 990-9.

13. Вuшк CM, Aшson DB. The genetic epidemiology of thinness. Obes Rev 2001; 2: 107-15.

14. Lee JH, ReEd DR, Price RA. Familial risk ratios for extreme obesity: implications for mapping human obesity genes. Int J Obes Relat Metab Disord 1997; 21: 935-40.

15. Katzmarzyk PT, Perusse L, Rao DC, Bouchard C. Familial risk of overweight and obesity in the Canadian population using the $\mathrm{WHO} / \mathrm{NIH}$ criteria. Obes Res 2000; 8: 194-7.

16. Khoury MJ, BeATy TH, Liang KY. Can familial aggregation of disease be explained by familial aggregation of environmental risk factors? Am J Epidemiol 1988; 127: 674-83.

17. Guo SW. Inflation of sibling recurrence-risk ratio, due to ascertainment bias and/or overreporting. Am J Hum Genet 1998; 63: 252-8.
18. Whitaker RC, Wright JA, Pepe MS, Seidel KD, Dietz WH. Predicting obesity in young adulthood from childhood and parental obesity. N Engl J Med 1997; 337: 869-73.

19. Yoon PW, Scheuner MT, Peterson-Oehike KL, Gwinn M, Faucett A, Khoury MJ. Can family history be used as a tool for public health and preventive medicine? Genet Med 2002; 4: 304-10.

20. Sнaм P. Statistics in Human Genetics. Series. John Wiley \& Sons. New York 1998.

21. Guo SW. Does higher concordance in monozygotic twins than in dizygotic twins suggest a genetic component? Hum Hered 2001; 51: 121-32.

22. Maes HH, Neale MC, Eaves LJ. Genetic and environmental factors in relative body weight and human adiposity. Behav Genet 1997; 27: 325-51.

23. Herskind AM, McGue M, Sorensen TI, Harvald B. Sex and age specific assessment of genetic and environmental influences on body mass index in twins. Int J Obes Relat Metab Disord 1996; 20: 10613.

24. Pietilainen KH, Kaprio J, Russanen A, Winter T, RIMPELA A, VIKEN RJ ET AL. Distribution and heritability of BMI in Finnish adolescents aged 16y and 17y: a study of 4884 twins and 2509 singletons. Int J Obes Relat Metab Disord 1999; 23: 107-15.

25. StunKard AJ, Foch TT, Hrubec Z. A twin study of human obesity. JAMA 1986; 256: 51-4.

26. Schousboe K, Visscher PM, ERbas B, Kyvik KO, HopPer JL, HenRIKSEN JE Et aL. Twin study of genetic and environmental influences on adult body size, shape, and composition. Int J Obes Relat Metab Disord 2004; 28: 39-48.

27. Schousboe K, WiLemsen G, Kyvik KO, Mortensen J, Boomsma DI, Cornes BK et aL. Sex differences in heritability of BMI: a comparative study of results from twin studies in eight countries. Twin Res 2003; 6: 409-21.

28. Neison TL, Brandon DT, Wiggins SA, Whitfield KE. Genetic and environmental influences on bodyfat measures among African-American twins. Obes Res 2002; 10: 733-9.

29. Samaras K, Spector TD, Nguyen TV, Baan K, CampbeLl LV, Keluy PJ. Independent genetic factors determine the amount and distribution of fat in women after the menopause. J Clin Endocrinol Metab 1997; 82: 781-5.

30. Boomsma D, Busjahn A, Peltonen L Classical twin studies and beyond. Nat Rev Genet 2002; 3: 872-82. 
31. Korkeila M, Kaprio J, RISSANen A, Koskenvuo M. Effects of gender and age on the heritability of body mass index. Int J Obes 1991; 15: 647-54.

32. Cardon LR, Carmew D, Fabsitz RR, Reed T. Genetic and environmental correlations between obesity and body fat distribution in adult male twins. Hum Biol 1994; 66: 465-79.

33. FabSTIZ RR, CARMEU D, HewitT JK. Evidence for independent genetic influences on obesity in middle age. Int J Obes Relat Metab Disord 1992; 16: 657-66.

34. Awison DB, Heshra S, Neale MC, Lykken DT, HEYMSFIELD SB. A genetic analysis of relative weight among 4,020 twin pairs, with an emphasis on sex effects. Health Psychol 1994; 13: 362-5.

35. Stunkard AJ, Harris JR, Pedersen NL, Mcciearn GE. The body-mass index of twins who have been reared apart. N Engl J Med 1990; 322: 1483-7.

36. Auison DB, Kaprio J, Korkeila M, Koskenvuo M, Neale MC, Hayakawa K. The heritability of body mass index among an international sample of monozygotic twins reared apart. Int J Obes Relat Metab Disord 1996; 20: 501-6.

37. Bouchard C, TREMBLAY A. Genetic influences on the response of body fat and fat distribution to positive and negative energy balances in human identical twins. J Nutr 1997; 127: 943S-947S.

38. Stunkard AJ, Sorensen TI, Hanis C, Teasdale TW, Chakraborty R, Schull WJ et al. An adoption study of human obesity. N Engl J Med 1986; 314: 193-8.

39. Botstein D, Risch N. Discovering genotypes underlying human phenotypes: past successes for mendelian disease, future approaches for complex disease. Nat Genet 2003; 33 Suppl: 228-37.

40. Altmuler J, Palmer LJ, Fischer G, Scherb H, Wist M. Genomewide scans of complex human diseases: true linkage is hard to find. Am J Hum Genet 2001; 69: 936-50.

41. LANDER ES, Schork NJ. Genetic dissection of complex traits. Science 1994; 265: 2037-48.

42. Haseman JK, Elston RC. The investigation of linkage between a quantitative trait and a marker locus. Behav Genet 1972; 2: 3-19.

43. Gнosн S, Cowns FS. The geneticist's approach to complex disease. Annu Rev Med 1996; 47: 333-53.

44. LANDER E, KRUglyak L Genetic dissection of complex traits: guidelines for interpreting and reporting linkage results. Nat Genet 1995; 11: 241-7.

45. DamcotT CM, SACK P, SHuLdiner AR. The genetics of obesity. Endocrinol Metab Clin North Am 2003; 32: 761-86.
46. Chen W, L S, Cook NR, RosNer BA, SRinivasan SR, BOERWINKIE E ET AL. An autosomal genome scan for loci influencing longitudinal burden of body mass index from childhood to young adulthood in white sibships: The Bogalusa Heart Study. IntJ Obes Relat Metab Disord 2004; 28: 462-9.

47. Arya R, Duggiraia R, Jenkinson CP, Aimasy L, Biangero J, O'CONNEL P ET AL. Evidence of a novel quantitativetrait locus for obesity on chromosome $4 \mathrm{p}$ in Mexican Americans. Am J Hum Genet 2004; 74: 272-82.

48. Li WD, Dong C, Li D, Zhao H, Price RA. An obesity-related locus in chromosome region 12q23-24. Diabetes 2004; 53: 812-20.

49. Lu YJ, Xu FH, Shen H, Lu YZ, Deng HY, Zhao LJ ET AL. A follow-up linkage study for quantitative trait loci contributing to obesity-related phenotypes. J Clin Endocrinol Metab 2004; 89: 875-82.

50. Meyre D, Lecoeur C, Delplanque J, Francke S, Vatin $\mathrm{V}$, DuRand E et al. A genome-wide scan for childhood obesity-associated traits in French families shows significant linkage on chromosome 6q22.31-q23.2. Diabetes 2004; 53: 803-11.

51. Colaku A, Rankinen T, Rice T, Leon AS, Rao DC, SKINNER JS ET AL. A genome-wide linkage scan for dietary energy and nutrient intakes: the Health, Risk Factors, Exercise Training, and Genetics (HERITAGE) Family Study. Am J Clin Nutr 2004; 79: 881-6.

52. Gulcher JR, Kong A, Stefansson K. The role of linkage studies for common diseases. Curr Opin Genet Dev 2001; 11: 264-7.

53. Bouchard C, Pérusse L, Rice T, Rao DC. Genetics of human obesity. Marcel Dekker, Inc. New York 2004. 157-200.

54. Albala C, Santos JL, Cifuentes M, Viliarroel AC, LeRA L, LBBerman C ET AL. Intestinal FABP2 A54T polymorphism: association with insulin resistance and obesity in women. Obes Res 2004; 12: 340-5.

55. Martínez JA, Corbalán MS, Sánchez-Viluegas A, Forga L, Marti A, MartínEz-GonZÁLEZ MA. Obesity risk is associated with carbohydrate intake in women carrying the Gln27Glu beta2-adrenoceptor polymorphism. J Nutr 2003; 133: 2549-54.

56. RedDEN DT, AшSON DB. Nonreplication in genetic association studies of obesity and diabetes research. J Nutr 2003; 133: 3323-6.

57. Page GP, George V, Go RC, Page PZ, Auson DB. "Are we there yet?": Deciding when one has demonstrated specific genetic causation in complex diseases and quantitative traits. Am J Hum Genet 2003; 73: 711-9. 
58. Marchini J, Cardon LR, Phimips MS, Donneluy P. The effects of human population structure on large genetic association studies. Nat Genet 2004; 36: 512-7.

59. ScHAID DJ. Transmission disequilibrium, family controls and great expectations. Am J Hum Genet 1998; 63: 935-41.

60. Santos JL, Pérez F, Carrasco E, Albala C. Uso de trios caso-padres en estudios epidemiológicos de asociación entre polimorfismos genéticos y enfermedades complejas. Rev Méd Chile 2002; 130: 1307-15.

61. Martin ER, Monks SA, Warren LL, Kaplan NL. A test for linkage and association in general pedigrees: the pedigree disequilibrium test. Am J Hum Genet 2000; 67: 146-54.

62. ScHAID DJ. Likelihoods and TDT for the caseparents design. Genet Epidemiol 1999; 16: 250-60.

63. Santos JL, Pérez-Bravo F, Carrasco E, Calviláa M, Albala C. Association between HLA-DQB1 alleles and type 1 diabetes in a case-parents study conducted in Santiago, Chile. Am J Epidemiol 2001; 153: 794-8.

64. Awson DB. Transmission-disequilibrium tests for quantitative traits. Am J Hum Genet 1997; 60: 676-90.

65. WaLdman ID, Robinson BF, Rowe DC. A logistic regression based extension of the TDT for continuous and categorical traits. Ann Hum Genet 1999; 63: 329-40.

66. Zolner S, Wen X, Hanchard NA, Herbert MA, Ober C, PRITCHARD JK. Evidence for extensive transmission distortion in the human genome. Am J Hum Genet 2004; 74: 62-72.

67. Camarena B, Santiago H, Aguilar A, Ruvinskis E, GonZÁLEZ-BARRANCO J, Nicolni H. Family-based association study between the monoamine oxidase A gene and obesity: implications for psychopharmacogenetic studies. Neuropsychobiology 2004; 49: 126-9.

68. Cassell PG, Saker PJ, Huxtable SJ, Kousta E, JaCKSON AE, HATTERSLey AT et aL. Evidence that single nucleotide polymorphism in the uncoupling protein 3 (UCP3) gene influences fat distribution in women of European and Asian origin. Diabetologia 2000; 43: 1558-64.

69. Delplanque J, Vasseur F, Durand E, Abderrahmani A, DiNA C, WAEBER G ET AL. Mutation screening of the urocortin gene: identification of new single nucleotide polymorphisms and association studies with obesity in French Caucasians. J Clin Endo- crinol Metab 2002; 87: 867-9.

70. Gelier F, Reichwald K, Dempfie A, Iwg T, Volmert C, HERPERTZ S ET AL. Melanocortin-4 receptor gene variant I103 is negatively associated with obesity. Am J Hum Genet 2004; 74: 572-81.

71. HinNey A, Lentes KU, Rosenkranz K, Barth N, Roth $\mathrm{H}$, ZiegleR A ET AL. Beta 3-adrenergic-receptor allele distributions in children, adolescents and young adults with obesity, underweight or anorexia nervosa. Int J Obes Relat Metab Disord 1997; 21: 224-30.

72. Hinney A, Bornscheuer A, Depenbusch M, Mierke B, Tole A, Middeke $\mathrm{K}$ et al. No evidence for involvement of the leptin gene in anorexia nervosa, bulimia nervosa, underweight or early onset extreme obesity: identification of two novel mutations in the coding sequence and a novel polymorphism in the leptin gene linked upstream region. Mol Psychiatry 1998; 3: 539-43.

73. Hinney A, Schneider J, Ziegler A, LehMkuhl G, POUSTKA F, SCHMIDT MH ET AL. No evidence for involvement of polymorphisms of the dopamine D4 receptor gene in anorexia nervosa, underweight, and obesity. Am J Med Genet 1999; 88: 594-7.

74. Hinney A, Hohmann S, Geuer F, Vogel C, Hess C, WERMTER AK ET AL. Melanocortin-4 receptor gene: case-control study and transmission disequilibrium test confirm that functionally relevant mutations are compatible with a major gene effect for extreme obesity. J Clin Endocrinol Metab 2003; 88: 4258-67.

75. Huang QY, Shen H, Deng HY, Conway T, Davies KM, L JL ET AL. Linkage and association of the CA repeat polymorphism of the IL6 gene, obesityrelated phenotypes, and bone mineral density (BMD) in two independent Caucasian populations. J Hum Genet 2003; 48: 430-7.

76. Le Stunff C, Fauin D, Bougneres P. Paternal transmission of the very common class I INS VNTR alleles predisposes to childhood obesity. Nat Genet 2001; 29: 96-9.

77. L WD, L D, Wang S, Zhang S, Zhao H, Price RA. Linkage and linkage disequilibrium mapping of genes influencing human obesity in chromosome region 7q22.1-7q35. Diabetes 2003; 52: 1557-61.

78. Long JR, Lu PY, Lu YJ, Lu Y, Xiong DH, EIZE L ET AL. APOE and TGF-beta1 genes are associated with obesity phenotypes. J Med Genet 2003; 40: 918-24. 
79. Roth H, Hinney A, Ziegler A, Barth N, Gerber G, StEIN K ET AL. Further support for linkage of extreme obesity to the obese gene in a study group of obese children and adolescents. Exp Clin Endocrinol Diabetes 1997; 105: 341-4.

80. Roth H, Korn T, Rosenkranz K, Hinney A, Ziegler A, KUnZ J ET AL. Transmission disequilibrium and sequence variants at the leptin receptor gene in extremely obese German children and adolescents. Hum Genet 1998; 103: 540-6.

81. Schauble N, Geler F, Siegfried W, Goldschmidt $H$, REMSChMidt H, HinNey A et al. No evidence for involvement of the promoter polymorphism -866 G/A of the UCP2 gene in childhood-onset obesity in humans. Exp Clin Endocrinol Diabetes 2003; 111: 73-6.

82. Wang HJ, Geluer F, Dempfie A, Schauble N, Friedel S, LICHTNER P ET AL. Ghrelin receptor gene: identification of several sequence variants in extremely obese children and adolescents, healthy normal- weight and underweight students, and children with short normal stature. J Clin Endocrinol Metab 2004; 89: 157-62.

83. Wang JG, He X, Wang GL, L Y, Zhou HF, Zhang WZ ET AL. Family-based associations between the angiotensin- converting enzyme insertion/deletion polymorphism and multiple cardiovascular risk factors in Chinese. J Hypertens 2004; 22: 48791.

84. Heude B, Dubois S, Charles MA, Deweirder M, Dina C, BoRY S JM ET AL. VNTR polymorphism of the insulin gene and childhood overweight in a general population. Obes Res 2004; 12: 499504.

85. Jiang Y, WiLk JB, Borecki I, Wimamson S, Destefano $\mathrm{AL}, \mathrm{XU} \mathrm{G}$ ET AL. Common variants in the $5^{\prime}$ region of the leptin gene are associated with body mass index in men from the National Heart, Lung, and Blood Institute Family Heart Study. Am J Hum Genet 2004; 75: 220-30. 